\title{
TRATAMIENTO DE LAS DEFINICIONES DE LOS CONECTORES DISCURSIVOS EN LA LEXICOGRAFÍA DIDÁCTICA CUBANA. EL CASO DEL DICCIONARIO BÁSICO ESCOLAR
}

\section{PROCESSING OF THE DEFINITIONS OF DISCURSIVE CONNECTORS IN CUBAN DIDACTIC LEXICOGRAPHY. THE CASE OF THE DICCIONARIO BÁSICO ESCOLAR}

\author{
Kelly Linares \\ Instituto de Literatura y Lingüistica "José Antonio Portuando Valdor" \\ klinares89@gmail.com
}

\begin{abstract}
RESUMEN
$\mathrm{El}$ interés que despiertan los marcadores del discurso (MD) dentro del proceso de enseñanza-aprendizaje de lenguas; el escaso análisis de su tratamiento en los diccionarios escolares hispanoamericanos y la carencia de investigaciones lexicográficas sobre $\mathrm{MD}$ en Cuba han motivado la realización de la presente investigación que tiene como objetivo principal examinar los tipos de definición empleados para un grupo de $\mathrm{MD}$, los conectores, en un diccionario escolar cubano. En él se repasan las características semántico-pragmáticas, señaladas por autores de referencia como Portolés (1999, 2001), Martín Zorraquino (1999, 2004), Fuentes (1996, 2003, 2009), Montolío (2001), de cada conector registrado en el diccionario con la finalidad de valorar si se incluyen o no en las definiciones objeto de análisis. Además, se evalúa en qué medida se ajusta el tratamiento de los conectores al desiderátum teórico de que como unidades léxicas con significado de procesamiento sus definiciones han de ser funcionales o explicativas.
\end{abstract}

Palabras clave: marcadores del discurso, conectores, diccionarios escolares, definición, lexicografía, pragmática

\begin{abstract}
The interest aroused by discourse markers (MD) within the process of teaching--learning languages; the scant analysis of its treatment in Spanish-American school dictionaries and the lack of lexicographical research on $\mathrm{MD}$ in Cuba have motivated the realization of the present investigation whose main objective is to examine the definitional types used for a group of $\mathrm{MD}$, the connectors, in a Cuban school dictionary. It reviews the semanticpragmatic characteristics, identified by reference authors such as Portolés (1999, 2001), Martín Zorraquino (1999, 2004), Fuentes (1996, 2003, 2009), Montolío (2001), of each connector registered in the dictionary in order to assess whether or not they are included in the definitions analysis. In addition, it is evaluated to what extent the treatment of the connectors is adjusted to the theoretical desideratum that as lexical units with processing meaning their definitions must be functional or explanatory.
\end{abstract}

Keywords: discourse markers, connectors, school dictionaries, definition, lexicography, pragmatics 


\section{INT'RODUCCIÓN}

A partir de los años sesenta y, fundamentalmente, en la década del 70 se desarrollan disciplinas lingüísticas como la lingüística del texto, la pragmática y el análisis del discurso, que no tienen la oración como nivel último analizable, sino que extienden su campo de análisis a niveles supraoracionales como el texto, el discurso, la conversación. Para López Serena y Borreguero (2010), a partir de la aparición de estas disciplinas se produce un salto desde el estudio inmanentista de la lengua hasta el pragmáticodiscursivo: "una necesaria inflexión desde la abstracción excesiva del sistema y la insuficiencia de la oración como nivel superior de análisis a la variación y organización propia del uso comunicativo" (2010, p. 382).

Por consiguiente, unidades lingüísticas como los marcadores del discurso (MD), que eran desterradas de los estudios gramaticales de la oración, fueron analizadas a partir de los diversos aparatos teóricos y metodológicos propuestos por las disciplinas antes mencionadas.

Los diversos estudios sobre los MD se han ocupado de su definición, clasificación, descripción y tratamiento lexicográfico. Esta última labor se ha dificultado por varios motivos, entre ellos: a) la pertenencia de estas unidades lingüísticas a diferentes categorías gramaticales; b) su heterogeneidad terminológica ligada a sus diversos abordajes teóricos; c) la complejidad de su clasificación funcional ${ }^{1}$; d) la naturaleza instruccional o de procesamiento de su significado ${ }^{2}$; e) su configuración prosódica y movilidad posicional (Martín Zorraquino, 2004, pp. 53-55; Vázquez, 2000). Sin

\footnotetext{
${ }^{1}$ Su clasificación funcional ha sido tratada, por una parte, desde una perspectiva onomasiológica (a partir de la existencia de diversos actos de habla, los MD se adscriben a funciones textuales vinculadas a estos, por ejemplo, advertencia, refutación, énfasis, etc.) y, por otra, semasiológica (a partir de la caracterización morfosintáctica, semántica y pragmática de los MD se crean grupos según sus equivalencias funcionales en el discurso, lo cuales permiten "determinar, con mejores garantías, las regularidades del comportamiento lingüístico de las unidades acotadas” (Martín Zorraquino, 2004, p. 54).

${ }^{2}$ Consiste en "una serie de instrucciones semánticas que guían las inferencias que se han de efectuar de los distintos miembros del discurso en los que aparecen estas unidades" (Martín Zorraquino y Portolés, 1999, p. 4071). Si bien la división realizada por Blakemore (1987) entre significado conceptual y procedimental ha recibido críticas pues las palabras con significado conceptual pueden, también, orientar el procesamiento de los enunciados y algunos MD, aun cuando presentan significado procedimental, conservan, en parte, un significado conceptual (Portolés [1998] 2001).
} 
embargo, a pesar de estas dificultades, se pondera la importancia de la aparición de los MD en la nomenclatura de los diccionarios y la necesidad de su adecuado tratamiento.

El auge de investigaciones lexicográficas de los MD en el ámbito hispánico, fundamentalmente en España, no se produce hasta la década del 90 del pasado siglo. Los acercamientos teóricos, descriptivos y lexicográficos al tema facilitaron y permitieron la elaboración de diccionarios específicos que sistematizan la información disponible sobre $\mathrm{MD}$ en artículos lexicográficos. Entre estos se encuentran el Diccionario de partículas de Santos Río (2003), el Diccionario de partículas discursivas del español (DPDE), coordinado por Briz, Portolés y Pons (2008), el Diccionario de conectores y operadores del español de Fuentes (2009) y, más recientemente, el Diccionario de Marcadores Discursivos para Estudiantes de Español como Lengua Extranjera (DIMAELE) de Holgado (2018). Los niveles de especialización de estos repertorios léxicos (que se traslucen en el mayor o menor empleo de metalenguaje, en los contenidos de los artículos lexicográficos y su grado de formalización y condensación) difieren en correspondencia con su función, determinada por el público meta y los propósitos perseguidos por los diccionaristas (finalidad), por ejemplo: realizar un diccionario para la codificación de mensajes o para su interpretación o descodificación.

A la proliferación de estudios, que se ocupan del tratamiento lexicográfico de los MD en diccionarios tipológicamente diferentes, y a la creación de diccionarios especializados se suma el interés por la enseñanza de este tipo de unidades lingüísticas en las clases de lenguas, tanto de primeras como de segundas. En relación con su legitimidad en las clases de español como lengua extranjera (E/LE), destaca Martín Zorraquino que los MD cumplen diversas funciones discursivas; forman parte de la competencia comunicativa de los hablantes; son palabras frecuentes con acepciones diversas, por lo tanto, necesarias para la construcción del discurso y la expresión de la subjetividad del hablante, y los errores que los aprendices de L2 cometen en su uso demuestran la impericia en el dominio de la lengua que se desea manejar como hablante nativo (Martín Zorraquino, 2004, pp. 56-57). Debemos añadir que esta impericia en su uso la demuestran no solo los aprendices de E/LE sino también los estudiantes de 
español como lengua materna (E/LM), que hacen una pobre utilización de ellos (Vázquez, 2008).

Dentro del proceso de enseñanza-aprendizaje de lenguas los diccionarios se erigen como una herramienta fundamental para el estudio de los MD. Por lo tanto, es importante que estas unidades lingüísticas aparezcan y sean tratadas de forma adecuada no solo en los diccionarios especializados, sino también en los generales de la lengua y en los didácticos, aun cuando los fines de estos repertorios lexicográficos son diferentes debido sus distintas naturalezas.

Los acercamientos a los MD desde una perspectiva metalexicográfica son numerosos en la parcela de la enseñanza de E/LE, según la bibliografía consultada. Sin embargo, el panorama resulta más desolador en la de E/LM, pues se ha examinado escasamente el tratamiento que los diccionarios escolares hispanoamericanos dan a los MD.

Por otra parte, en Cuba son escasos los trabajos sobre MD. Estos derivan, fundamentalmente, de proyectos de investigación como el "Proyecto de estudio de la norma lingüística culta hispánica Juan M. Lope Blanch”, y "Habana, Español Coloquial” (Hab.Es.Co, 2012). Han estado dirigidos, fundamentalmente, hacia la descripción del comportamiento de MD en el habla culta o coloquial de la variedad cubana del español. Destacan los acercamientos de Perdomo (2013), González Mafud y Perdomo (2014), Martínez Abreu (2015) y García Roche (2018).

El panorama presentado (el interés que despiertan los MD dentro del proceso de enseñanza-aprendizaje de lenguas; el escaso análisis de su tratamiento en los diccionarios escolares hispanoamericanos y la carencia de investigaciones lexicográficas sobre MD en Cuba) ha motivado la realización de esta investigación que se centrará en el examen de los tipos definición empleados para un grupo de $\mathrm{MD}$, los conectores, en un diccionario escolar cubano: Diccionario básico escolar (DBE) del Centro de Lingüística Aplicada de Santiago de Cuba (2014), dirigido por Miyares Bermúdez.

Se ha optado por el estudio de la definición por ser un elemento medular en el artículo lexicográfico y uno de los que comporta mayor dificultad en su elaboración, sobre todo en los diccionarios escolares, si se tiene en cuenta que sus destinatarios son 
hablantes nativos que se encuentran en un momento de aprendizaje y afianzamiento de la lengua materna.

La presente investigación se inserta dentro de los estudios sobre MD desde la perspectiva de la lexicografía aplicada a la didáctica. Se aproxima a estas unidades lingüísticas, específicamente a los conectores, desde un enfoque semántico-pragmático a partir de los estudios teóricos desarrollados, fundamentalmente, por los hispanistas Portolés (1999, 2001) y Martín Zorraquino (1999, 2004), aunque no se desatienden los aportes al tema de otros estudiosos como Fuentes (1996, 2003, 2009) o Montolío (2001). Para el análisis lexicográfico se siguen, principalmente, los postulados teóricos de Porto Dapena $(2002,2014)$.

\section{SOBRE LOS MARCADORES DEL DISCURSO: LOS CONECTORES}

El término marcador del discurso, popularizado por la autora norteamericana Schiffrin (1987) en su libro Discourse markers, es de los más empleados en los estudios lingüísticos hispánicos. En los diferentes abordajes investigativos del tema se constata que este término coexiste con otros, por ejemplo, con el término conector, en una relación sinonímica o es tomado como un hiperónimo "referido a casi cualquier clase de palabras sin significado proposicional” (Pons, 1998, s/p). En esta investigación, siguiendo a Martín Zorraquino y Portolés (1999), se adopta el término marcador del discurso como hiperónimo, pues se coincide con estos autores cuando declaran:

Sólo una parte de los marcadores conectan, no lo hacen, por ejemplo, los operadores argumentativos y mucho menos los 'marcadores conversacionales'. Ciertamente, los marcadores pueden relacionar una oración con otras unidades externas a ella, pero también es frecuente que relacionen unidades intraoracionales o unidades que pertenecen a categorías sintagmáticas distintas de la oración. Los marcadores, en fin, cohesionan el discurso, pero esta es sólo una de sus posibles propiedades (1999, p. 4057, nota al pie 6).

Por lo tanto, términos como conector y operador se refieren en este trabajo a tipos de $\mathrm{MD}^{3}$. Asimismo se adopta que:

[Los] marcadores del discurso son unidades lingüísticas invariables, no ejercen una función sintáctica en el marco de la predicación oracional - son, pues, elementos

\footnotetext{
${ }^{3}$ Para consideraciones diferentes a las adoptadas en este trabajo sobre los términos marcador del discurso, conector y operador discursivo, véanse Pons (1998) y Fuentes (2003).
} 
marginales-y poseen un cometido coincidente en el discurso: el de guiar, de acuerdo con sus distintas propiedades morfosintácticas, semánticas y pragmáticas, las inferencias que se realizan en la comunicación (Martín Zorraquino y Portolés, 1999, p. 4057).

Los autores antes citados, a partir de una propuesta de clasificación que se basa en buscar para el marcador un significado unitario que dé cuenta de todos sus usos, distinguen cinco tipos de MD:

1. estructuradores de la información: pues, en primer lugar/en segundo lugar, por cierto, a propósito, entre otros.

2. conectores: además, encima, en consecuencia, entonces, sin embargo, entre otros.

3. reformuladores: es decir, de todos modos, en conclusión, en fin, entre otros.

4. operadores argumentativos: en el fondo, de hecho, por ejemplo, en particular, entre otros.

5. marcadores conversacionales: bueno, claro, hombre, mira, eh, entre otros.

\subsection{LOS CONECTORES}

Es de interés para este trabajo el grupo de los conectores. Si bien autores como Gili y Gaya (1943), Pons (1998), Portolés ([1998] 2001), Briz (1993) reconocen que las conjunciones coordinantes (y, pero, sino) y la subordinante aunque son los conectores prototípicos — siguiendo las palabras de Pons (1998)—, en este estudio no se incluyen dentro del inventario de los MD porque, aunque vinculan semánticamente dos miembros del discurso y orientan el proceso inferencial, no cumplen con todas la características morfosintácticas que se han adoptado para distinguir los MD, entre ellas:

1. Son unidades lingüísticas invariables (no conservan la capacidad de flexión y de combinación de su miembros. Por tal razón por consiguiente es un MD y no así por este motivo):

(1) a. Juan está muy ocupado y, por consiguiente, no te podrá acompañar al teatro.

b. Juan está muy ocupado y, por este motivo, no te podrá acompañar al teatro.

(2) a. *Juan está muy ocupado. Tiene que entregar un informe, leer los trabajos de los alumnos y evaluarlos. Por consiguientes, no te podrá acompañar al teatro.

b. Juan está muy ocupado. Tiene que entregar un informe, leer los trabajos de los alumnos y evaluarlos. Por estos motivos, no te podrá acompañar al teatro.

2. Se deben a un proceso de gramaticalización, aunque no todos presentan el mismo grado de gramaticalización. Señalan Martín Zorraquino y Portolés 
(1999, p. 4060) que "los marcadores del discurso proceden de la evolución de una serie de sintagmas que, de una parte, van perdiendo sus posibilidades de flexión y combinación, y, de otra, van abandonado su significado conceptual y se especializan en otro de procesamiento".

3. Presentan movilidad sintáctica ${ }^{4}$.

(3) a. Está muy callada. Por cierto, ¿le comentaste sobre aquel asunto?

b. Está muy callada. ¿Le comentaste, por cierto, sobre aquel asunto?

c. Está muy callada. ¿Le comentaste sobre aquel asunto?, por cierto.

4. No modifican ningún elemento dentro de la sintaxis oracional.

(4) Lo puso todo encima de la mesa.

Encima funciona como adverbio de lugar que modifica al verbo.

(5) Llegas tarde a clases y, encima, no haces la tarea.

Encima funciona como marcador del discurso (conector).

5. No reciben especificadores ni adyacentes complementarios ${ }^{5}$.

(6) a. En todo caso, vive lejos.

b. *En todo caso de esos, vive lejos.

c. *Muy en todo caso, vive lejos (Martín Zorraquino y Portolés, 1999, p. 4066)

6. No pueden ser negados.

(7) a. No vino, pues, Iuan a la fiesta, sino Antonio.

b. No vino, pues, Juan a la fiesta, sino que se quedó en casa.

c. No vino, pues, Juan a la fiesta, sino que ya estaba.

d. No vino, pues, Juan a la fiesta, sino a la cena de después. 4067).

e. *No vino, pues, Juan a la fiesta, sino por tanto (Martín Zorraquino y Portolés, 1999, p.

7. No se coordinan entre sí (por ejemplo, *además y sin embargo.)

Martín Zorraquino y Portolés (1999) para definir los conectores tienen en cuenta las instrucciones argumentativas de su significado. Son los MD que "vinculan semántica y pragmáticamente un miembro del discurso con otro anterior, de tal forma que el

\footnotetext{
${ }^{4}$ Esta característica diferencia a los MD de las conjunciones de la gramática tradicional porque, aun cuando la noción de conexión les es intrínseca (Pons, 1998), estas siempre anteceden al sintagma que introducen (Fuentes, 1996; Martín Zorraquino y Portolés, 1999). Dicha razón hace que conjunciones como pero, $y$, o aunque queden fuera de nuestro estudio.

${ }^{5}$ Excepto los MD además, encima y aparte, que pueden recibir complementos con de (Martín Zorraquino y Portolés, 1999, p. 4066).
} 
marcador guía las inferencias que se han de efectuar del conjunto de los dos miembros discursivos conectados" (1999, p. 4080). La progresión discursiva no depende de la capacidad de los conectores para unir informaciones sino de su capacidad para orientar y restringir el proceso argumentativo del discurso.

Es por ello que solo incluyen dentro del grupo de los conectores los MD que tienen instrucciones de conexión y argumentativas en su significado. Por lo tanto, excluyen los temporales y ordenadores discursivos (Fuentes, 1996), los organizadores de la información (Montolío, 2001) y los reformulativos (Fuentes, 1996); los cuales no realizan una operación argumentativa y se limitan a actuar en el marco informativo, aun cuando tienen carácter fórico.

En esta investigación se asume que los conectores son MD que cohesionan dos miembros del discurso y actúan, debido a las instrucciones argumentativas de su significado, sobre las inferencias que han de efectuarse de los miembros que relacionan, al condicionar la aparición de unas inferencias y la negación de otras. En un ejemplo como:

(8) Luis es muy inteligente; sin embargo, suspendió el examen.

Los enunciados están antiorientados argumentativamente: el primero (Luis es muy inteligente) orienta hacia unas conclusiones contrarias a la que aparece en el segundo (suspendió el examen). Estos enunciados antiorientados se relacionan a través del conector sin embargo, el cual por su significado indica que contraria a la conclusión que se puede esperar del enunciado es muy inteligente, Luis suspendió el examen.

Se adopta en este trabajo la clasificación de los conectores de Martín Zorraquino y Portolés (1999), quienes los dividen en tres grupos de acuerdo con su significado:

1. Conectores aditivos: unen a un miembro discursivo anterior otro con su misma orientación argumentativa. De esta forma, posibilitan la inferencia de conclusiones que serían difíciles de obtener si los dos miembros permaneciesen independientes (1999, pp. 4093-4099).

(9) Tiene unas condiciones físicas increíbles y, además, es muy alta. Puede jugar en el equipo. 
2. Conectores consecutivos: Presentan el miembro discursivo en el que se encuentran como una consecuencia del miembro anterior. (1999, pp. 4099-4109).

(10) Este sector económico empieza a entrar en crisis; por tanto, es el momento de diversificar nuestras inversiones (Montolío, 2001, p. 100).

3. Conectores contraargumentativos: unen dos miembros del discurso, de forma que el segundo suprime o atenúa alguna conclusión que pueda inferirse del primero (1999, pp. 4109-4121).

(11) Los críticos consideran que su libro es excelente; sin embargo, sus niveles de venta son bajos.

\section{SOBRE EL DICCIONARIO BÁSICO ESCOLAR Y LA SELECCIÓN DE LA}

\section{MUEST'RA}

En Cuba la lexicografía didáctica (LD) ${ }^{6}$ desde una perspectiva práctica se ha desarrollado, principalmente, a partir de los estudios que se realizan en el Centro de Lingüística Aplicada (CLA) de Santiago de Cuba.

Entre los años 1990 y 1995 se llevó a cabo en el CLA el proyecto "Estudio del vocabulario activo-funcional del escolar cubano" ${ }^{7}$, dirigido por Miyares Bermúdez. Tuvo como finalidad "conocer el léxico de los educandos cubanos, así como el uso que hacen estos de las palabras, especialmente las comprendidas en la esfera conceptual - sustantivos, adjetivos, verbos y adverbios- sin dejar de analizar también las otras clases de palabras y las frases, locuciones y siglas presentes en el vocabulario de los niños” (Miyares Bermúdez et al., 2006, p. 7). La muestra estuvo integrada por textos orales y escritos, realizados por estudiantes de $2 .^{\circ}$ a $6 .^{\circ}$ grados y de 10 territorios del país. En total se recogieron 700000 palabras y 26251 palabras diferentes que son la base fundamental del Diccionario básico escolar (DBE, en sus diversas ediciones).

\footnotetext{
${ }^{6} \mathrm{La}$ LD es una disciplina, una rama de la lexicografía, vinculada con la elaboración de diccionarios para estudiantes. Propone Azorín (2000, p. 21) emplear "la denominación Diccionario didáctico como etiqueta genérica para designar a los diccionarios especialmente diseñados para los usuarios que se encuentran en el proceso de aprendizaje de una lengua". Estos se dividen en dos grandes grupos según sus objetivos y usuarios potenciales: diccionarios didácticos de español como lengua materna, para usuarios en fase de aprendizaje de la lengua materna, y diccionarios didácticos de español como lengua no materna, para usuarios en fase de aprendizaje de una segunda lengua (Hernández Hernández, 1989, 2003, 2008; Azorín, 2000). A los primeros se los denomina escolares y dentro de los segundos se distinguen los de aprendiraje (diccionarios monolingües para extranjeros), los bilingües y los semibilingües (diccionarios mixtos que combinan los bilingües con los monolingües).

${ }^{7}$ De este proyecto deriva la obra Léxico activo-funcional del escolar cubano (2006).
} 
El DBE es la obra más difundida de la lexicografía escolar en Cuba, muestra de ello son sus múltiples ediciones, reimpresiones, y su distribución en diversos formatos (papel, CD, internet, intranet nacional). Sus destinatarios son los escolares con edades comprendidas entre los 10 y los 18 años, es decir, los que se encuentran en segundo ciclo de la enseñanza primaria ( $4 .^{\circ}$ y $5 .^{\circ}$ grados), los de la enseñanza secundaria y los de la preuniversitaria y politécnica. Esta franja temporal resulta excesivamente amplia (Sánchez Muñoz, 2002-2003) y ha sido criticada por varios investigadores que han reseñando las diferentes ediciones de la obra (Forgas y Bargalló, 2005-2006, p. 254; Nomdedeu, 2012, pp. 245-246).

Uno de los aspectos que se echa en falta en la "Introducción" de esta obra es la declaración de las fuentes utilizadas para la conformación de su nomenclatura. En Ruiz Miyares y Tarp (2015) se declara que las 12000 entradas se seleccionaron del corpus Léxico activo-funcional del escolar cubano, de libros de texto de diferentes grados, de periódicos y revistas cubanas dirigidas a los jóvenes, así como de un corpus basado en un estudio sobre el uso de los afijos por parte de estudiantes de $7 .^{\circ}$ a $9 .^{\circ}$ grados. Sin embargo, no quedan claros los criterios seguidos para la selección de estos textos y de las unidades léxicas que componen no solo la macroestructura sino también la microestructura (unidades léxicas sublematizadas) del diccionario.

La nomenclatura de este diccionario en su cuarta edición, versión papel, constituye el corpus de este trabajo.

En primer lugar, para la selección de la muestra: los conectores presentes en el $D B E$, se elaboró un listado de referencia a partir de los conectores inventariados en el capítulo 63 de la Gramática descriptiva de la lengua española (Martín Zorraquino y Portolés, 1999). A saber:

1. Aditivos: además, encima, aparte, incluso, inclusive, por añadidura, es más.

2. Contraargumentativos: en cambio, por el contrario, al contrario, antes bien, sin embargo, no obstante, con todo, por contra, empero, ahora bien, ahora, eso sí.

3. Consecutivos: por (lo) tanto, por consiguiente, consiguientemente, consecuentemente, por ende, en consecuencia, de abi, entonces, pues, asi, asi pues. 
Una vez establecido este listado se procedió a su búsqueda en el DBE. Se tuvo en cuenta no solo la aparición de la forma (como lema o sublema) sino también que se recogiera con el significado de MD. Los resultados obtenidos fueron los siguientes:

1. Aditivos: además, encima.

2. Contraargumentativos: por el contrario, al contrario, sin embargo, con todo.

3. Consecutivos: por (lo) tanto, entonces, pues.

Estos conectores registrados en el $D B E$ constituyen la muestra de nuestra investigación ${ }^{8}$. Se debe aclarar que aparecen lematizadas otras formas como incluso, inclusive, ahora, así, pero no con el significado de conector discursivo, por lo tanto, no serán analizadas.

\section{ASPECTOS GENERALES SOBRE LAS DEFINICIONES DE LOS MARCADORES DEL DISCURSO}

Resulta recurrente la afirmación de que la tarea más ardua en el proceso de realización de un artículo lexicográfico de un MD es la elaboración de su definición (Vázquez, 2000; Martín Zorraquino, 2004; Sanmartín, 2010; Holgado, 2014).

Las definiciones sinonimicas de estas unidades léxicas han marcado el proceder de muchos diccionarios generales de lengua que no dan cuenta de los sentidos diversos de los MD, sino que en una entrada se limitan a ofrecer como significado otros MD que, o no son sinónimos del definido o no lo son en todos los contextos (Vázquez, 1995-1996). De igual forma, se incurre en el fenómeno de la circularidad, pues el uso de este tipo definición conduce a un recorrido de un MD a otro que actúa como definición y que a su vez es definido con el primer MD. El usuario que realiza la consulta culmina sin saber el significado de los MD buscados. También aparecen definiciones perifrásticas poco aclaratorias y deficientes y, en el peor de los casos, se llega a las pistas perdidas, pues aparece como definición de un MD otros que no se encuentran lematizados o sublematizados.

\footnotetext{
${ }^{8}$ La comparación con otras dos obras de similar naturaleza: el Diccionario del estudiante. Secundaria y Bacbillerato (RAE, [2005] 2011) y el Diccionario Secundaria y Bachillerato. Lengua Española (2003) de Ediciones $\mathrm{SM}$, dirigido por Maldonado, arroja que el $D B E$ ha incluido una cantidad menor de conectores. Por ejemplo, el Diccionario del estudiante... de la RAE del listado de referencia recoge los siguientes conectores: además, encima, incluso, por añadidura, en cambio, por el contrario, antes bien, sin embargo, no obstante, con todo, empero, ahora bien, ahora, por (lo) tanto, por consiguiente, consiguientemente, por ende, pues, así, así pues.
} 
Por esta razón, lo más recomendable es el empleo, para este tipo de unidades léxicas, de la definición lexicográfica formal, funcional o explicativa, es decir, la que se realiza en metalengua de signo y a través de la cual "en lugar del contenido, lo que se pone de manifiesto son características relativas a aspectos formales -funcionales, morfológicos, sintácticos o combinatorios, pragmáticos, etc.- de la palabra utilizada como definiendum" (Porto Dapena, 2014, p. 61). Esta debe ser empleada en la descripción de las palabras gramaticales, en las que predomina el significado procedimental y las que, por tanto, no se dejan someter a la ley de la sinonimia, que consiste en la "exigencia de que el definiente esté constituido por una forma adecuada a la función sintáctica propia del definido" (Seco, 1987, p. 21).

Acertadamente expresa Portolés ([1998] 2001, p. 153) que "a diferencia de las definiciones del léxico conceptual ${ }^{9}$ la de los marcadores han de reflejar no lo que la palabra representa, sino lo que la fuerza en la comprensión del miembro del discurso en el que aparece o al que acompaña”. Aunque, como destaca Martín Zorraquino (2004, pp. 61-62), la dificultad que entraña construir este tipo de definición conduce al uso en muchas obras lexicográficas generales de la antes mencionada definición sinonímica.

Muchas de las deficiencias en la construcción de las definiciones de los MD han sido superadas en las obras lexicográficas especializadas. Por ejemplo, diccionarios de MD como el DPDE y el DIMAELE han optado por el uso de definiciones funcionales sencillas, sin tanta carga metalingüística, y han creado artículos lexicográficos que difieren de los que se encuentran en los diccionarios generales de lengua, pues contienen una mayor cantidad de apartados que ofrecen información sobre la prosodia, la sintaxis y los registros de uso de los MD.

Portolés ([1998] 2001, pp. 152-155) cuando se refiere al tipo de definición que debe emplearse para los MD presenta el modelo empleado en el DPDE y ejemplifica con la

\footnotetext{
${ }^{9}$ A las palabras con significado conceptual (sustantivos, adjetivos, verbos y algunos adverbios) le corresponde la definición conceptual que se formula en metalengua de contenido y en la que se expresa "en otras palabras de la misma lengua el contenido significativo o conceptual del definido" (Porto Dapena, 2002, p. 282). Esta puede ser sinonímica: el definidor es un sinónimo del definido o perifrástica: el definidor está constituido por una frase o sintagma (2002, p. 285).
} 
definición del conector sin embargo: "Presenta el miembro del discurso en el que aparece como una conclusión 'descriptor'. Esta conclusión es contraria a otra que se pudiera inferir a partir de un argumento anterior "diferenciador". Como vemos consta de un 'descriptor' que destaca la función discursiva que permite relacionar al MD con otros con la misma función, y de un 'diferenciador' que destaca lo que diferencia al MD de los otros que integran su mismo grupo.

A continuación analizaremos qué tipos de definiciones emplea el $D B E$ para los conectores de la muestra. Se hará un breve repaso de las características semánticopragmáticas de cada conector señaladas por algunas obras de referencia con el objetivo de valorar si se incluyen o no en las definiciones objeto de análisis. Además, se evaluará en qué medida se ajusta el tratamiento de los conectores al desiderátum teórico de que como unidades léxicas con significado de procesamiento sus definiciones han de ser funcionales o explicativas y no conceptuales.

\section{SOBRE LAS DEFINICIONES DE LOS CONECTORES EN EL DICCIONARIO BÁSICO ESCOLAR}

\subsection{EL CASO DE ADEMÁS}

Además es el conector de uso más frecuente dentro del grupo de los aditivos, así lo señalan autores como Fuentes (1996, p. 27), Martín Zorraquino y Portolés (1999, p. 4094), y Montolío (2001, p. 143). Apuntan Martín Zorraquino y Portolés (1999, p. 4094) que "vincula dos miembros del discurso con la misma orientación argumentativa ${ }^{10}$. El segundo facilita inferencias que sería difícil lograr únicamente del primero". Este segundo miembro, puede aumentar la fuerza argumentativa del primero y se presenta, por lo general, como más relevante (Montolío 2001, p. 157).

En el $D B E$ este conector se define como sigue:

(12) además adv. c. Significa también, aparte de.

La definición es sinonímica múltiple, pues se utilizan dos unidades léxicas como sinónimas del conector definido: también y aparte de. Por lo tanto, se emplea un tipo de

\footnotetext{
${ }^{10}$ Los miembros que vincula el conector aditivo además conducen a la misma conclusión.
} 
definición conceptual para definir una palabra con significado de procesamiento: recurso, como ya expresamos, no recomendable.

También aparece explícito el verbo cópula significa como introductor del enunciado que funciona como definición de tipo conceptual; uso que se documenta en diccionarios antiguos como el Tesoro de Covarrubias de 1611 (Llopis, 2006, p. 1087 y Porto Dapena, 2014, p. 26), pero no es propio de los diccionarios modernos, los cuales sistematizan la información lexicográfica prescindiendo del verbo cópula, el cual solo se hace explícito en las definiciones funcionales o explicativas introducidas por indica, presenta, introduce, sirve para, se usa para, etc.

Por un lado, el camino que impone la definición de además en el DBE nos lleva a la entrada también que en su segunda acepción se define de manera sinonímica como además. El escolar que se enfrente a la búsqueda del significado del conector de la muestra terminará en el mismo punto donde comenzó su pesquisa. Una vez más, el fenómeno de la circularidad se hace presente en la práctica lexicográfica.

A pesar de que estas unidades léxicas se ofrecen como sinónimas y tienen desde el punto de vista semántico el significado básico de 'añadir o sumar una información a la ya presentada o conocida con anterioridad', no son intercambiables en todos los contextos $^{11}$. Al respecto nos dice la Nueva gramática de la lengua española (NGLE, 2009, pp. 2362-2363):

En contra de agregar además al grupo de los adverbios focales, y a favor de considerarlo entre los conectores aditivos, está el hecho de que se construye con complementos preposicionales, sean expresos o tácitos [...]. El complemento preposicional (ya sea expreso, ya tácito), que caracteriza al adverbio además lo diferencia marcadamente de también, con el que no puede alternar en muchos contextos, en especial en los de foco nominal.

Por otro lado, la búsqueda de la otra unidad léxica que se pone como sinónimo (aparte de) nos lleva a la definición: 'indica omisión' que, aun cuando es funcional, resulta poco esclarecedora por su brevedad, y su significado no se corresponde con el del conector aditivo además.

\footnotetext{
11 Véase la comparación que realiza Cuartero (1995) de las unidades léxicas además y también y las consideraciones de Fuentes (1996, p. 29).
} 
Lo más llamativo es que en este caso se incurre en un error por omisión, porque el adverbio aparte sí funciona como conector aditivo (Martín Zorraquino y Portolés, 1999, p. 4096), pero el significado que tiene como tal no se registra en el DBE. De igual forma, no resultaría apropiado definir el conector discursivo más frecuente de los aditivos por remisión a uno que es semejante, pero menos frecuente.

Asimismo, la unidad léxica que se consigna como sinónimo es aparte de que, por su construcción, es afín con además de, combinación a la cual no se hace alusión en el artículo lexicográfico de además. Sobre estas combinaciones apuntan Martín Zorraquino y Portolés (1999) que, aun cuando permanecen con el mismo significado y la posición de inciso:

Pierden en estos casos su capacidad de conexión entre dos miembros del discurso, ya que el elemento al que se hacía referencia anafóricamente con el marcador es el que aparece ahora en el complemento: a. Es muy simpático y, además, cocina muy bien. b. Además de ser muy simpático, cocina muy bien. (p. 4066).

La remisión de una entrada a otra en la que no se encuentra el significado esperado, sino otra acepción de la palabra a la que se remite nos recuerda los errores en los que ha incurrido, otrora con mayor asiduidad que en la actualidad, la lexicografía didáctica hispana, porque la macroestructura de los diccionarios ha derivado, en algunos casos, de la reducción de obras lexicográficas mayores (diccionarios generales), lo que conduce a inconsistencias similares a la aquí presentada.

\subsection{EL CASO DE ENCIMA}

El conector aditivo encima es más frecuente en la lengua hablada, en registros informales y en escritos poco formales, según se declara en alguna bibliografía (Montolío, 2001, p. 158; DPDE, 2008).

$\mathrm{Al}$ igual que además vincula dos miembros del discurso con la misma orientación argumentativa, pero a diferencia de este indica que el miembro que lo precede es suficiente para arribar a determinada conclusión. Asimismo, el argumento que introduce aumenta la escala argumentativa del argumento anterior y refuerza "el sentido de exceso, importancia, abuso o sorpresa" (DPDE, 2008) que de él se desprende; lo que lo diferencia también de además. A ello se suma que la valoración de 
la información que se desprende de su uso es, por lo general, de carácter negativo (Fuentes, 1996, p. 27; Montolío, 2001, p. 158; NGLE, 2009: p. 2363). Al decir de Montolío (2001, p. 160), por este carácter subjetivizador y su relación con el registro oral espontáneo: "El uso de este conector no resulta frecuente —ni recomendable—en los textos que se presenten como objetivos [...], de hecho, en la lengua escrita, el uso de encima se restringe a los textos de opinión, es decir, aquellos en los que el género textual admite que el autor exprese su propia evaluación".

En el diccionario que nos ocupa se define el conector encima como se muestra a continuación:

(13) encima adv. $1[\ldots] 3$ Además, por si fuera poco.

En este caso, observamos que la definición es igualmente sinonímica. En primer lugar se remite al conector más frecuente de la serie de los aditivos además; lo que lleva, como vimos anteriormente, a la circularidad y a la obtención de un significado erróneo.

En segundo lugar, se da como significado una expresión (por si fuera poco) cuyo estatuto como conector aditivo vacila, pues no toda la bibliografía consultada la considera como tal. No se hace alusión a esta expresión en Fuentes (1996), Martín Zorraquino y Portolés (1999) y Portolés ([1998] 2001); Montolío (2001, p. 161) se refiere a ella como una secuencia que, junto a para colmo, presenta un valor semejante al del MD encima; y la NGLE (2009) la presenta como una "fórmula asimilable a las locuciones adverbiales" (2009, p. 2356) y como un "adverbio conector aditivo y de precisión y particularización" (2009, p. 2361) al igual que además, encima, asimismo, por añadidura, entre otros. En esta misma obra más adelante se explica que "encima y por si fuera poco añaden información que se sitúa en un punto superior de la línea argumentativa introducida en el texto que los precede" (2009, p. 2361), y que la interpretación de por si fuera poco es más literal que la de encima, "donde poco se interpreta como 'de peso argumentativo escaso e insuficiente"' (2009, p. 2361). El DPDE lo registra en la entrada de encima ${ }^{1}$ como partícula semejante. 
¿La vacilación puede deberse a que aún no está totalmente gramaticalizado y a que su comportamiento como MD todavía no es estable?, aunque llama la atención que las obras que lo registran como MD son las más actuales.

Se tome por si fuera poco como un sinónimo del definido o como una definición parafrástica - la ausencia de esta expresión como sublema en el DBE nos inclina hacia esta segunda opción-, las pistas que ofrece sobre el significado de encima son escasas.

\subsection{EL CASO DE SIN EMBARGO}

Sin embargo es un conector perteneciente al grupo de los contraargumentativos e indica que el argumento que introduce lleva a una conclusión contraria a la que se podía esperar del argumento anterior, que puede estar explícito o ser una suposición contextual (Martín Zorraquino y Portolés, 1999, p. 4115; Portolés [1998] 2001). Señala Montolío que este conector presenta un argumento más fuerte que el que lo precede, es decir, con mayor fuerza argumentativa, por lo que "inclina el signo de la conclusión en su misma dirección argumentativa" (2001, p. 51).

Aparece definido en el DBE como sigue:

(14) embargo $[\ldots] / /$ conj. adversativa. sin embargo. No obstante, a pesar de.

Nuevamente nos encontramos ante una definición sinonímica múltiple. El primer sinónimo que aparece como definidor no se sublematiza en el DBE. Por tanto, estamos ante una pista perdida, que nos dice poco o nada sobre el significado de sin embargo. La impropiedad en el uso del conector contraargumentativo no obstante como definición de sin embargo se manifiesta en varios aspectos que podemos sintetizar como sigue: no obstante no se sublematiza ni se define en el DBE (pista perdida); es un conector contraargumentativo que introduce un argumento fuerte, por lo tanto, es afín con sin embargo, pero su uso es menos frecuente y su significado no es totalmente idéntico (Martín Zorraquino y Portolés, 1999, p. 4116; Montolío, 2001, p. 63). 
El otro sinónimo que se consigna en la definición es la locución preposicional a pesar de que aparece sublematizada en la obra ${ }^{12}$. Lo incorrecto de su uso radica, por una parte, en el empleo de una definición de tipo conceptual sinonímico para el conector sin embargo y, por otra, en que no constituye un sinónimo del conector aludido. Montolío clasifica esta locución preposicional como un conector contraargumentativo integrado entonativa y sintácticamente en la oración y, además, introductor de un argumento débil, es decir, presenta un impedimento que no será un obstáculo para lo se expresa en la oración principal (Montolío, 2001, pp. 47-62). De igual forma, la definición que se ofrece de a pesar de brinda pocas luces sobre el significado de sin embargo. Véase a continuación:

(15) pesar [...] loc. conj. a pesar o a pesar de. Contra todos los obstáculos, no obstante.

\subsection{EL CASO DE CON TODO}

Este conector contraargumentativo es semejante a sin embargo y no obstante porque introduce un argumento que conduce a una conclusión contraria a la que se podía inferir del argumento anterior. La diferencia radica en que el miembro anterior se presenta como un argumento fuerte para arribar a una conclusión contraria a la que introduce el conector (Martín Zorraquino y Portolés, 1999, pp. 4116-4118; Montolío, 2001, pp. 70-74). Entre los refuerzos argumentativos que se emplean para hacer que el primer miembro discursivo sea fuerte se encuentran: "prolija acumulación de datos informativos; acopio de múltiples argumentos coorientados; uso de elementos léxicos, partículas y construcciones sintácticas con carácter intensificador, etc.” (Montolío, 2001, p. 74).

En el DPDE se señala su mayor frecuencia en el registro formal de la lengua. Este conector se define en el $D B E$ de la manera siguiente:

(16) todo, a [...] // loc. conj. con todo. Sin embargo, no obstante.

En este caso el uso de la definición sinonímica nos conduce, por las dos vías de búsqueda que impone la remisión, hacia la pista perdida no obstante. También nos lleva

${ }^{12}$ En el $D B E$ aparece con la marca gramatical correspondiente a la locución conjuntiva. Sobre la consideración de esta locución como preposicional o conjuntiva véase la NGLE (2009, pp. 2276-2284; 2460). 
a a pesar de que, como vimos, aun cuando tiene un sentido contraargumentativo, se comporta sintácticamente diferente a estos conectores y desde el punto de vista semántico-pragmático introduce argumentos débiles.

Nos hallamos ante una definición que no clarifica el significado del definido; que acuña como sinónimos conectores que, si bien son semejantes, no son totalmente idénticos, y que envuelve al usuario en una búsqueda que lleva a pistas perdidas.

\subsection{EL CASO DE AL CONTRARIO, POR EL CONTRARIO}

Este par de conectores ha sido tratado de diversas maneras en la bibliografía consultada. Algunos diccionarios los registran como sinónimos (Diccionario de la lengua española, DLE, 2014), otros, como variantes (Diccionario del estudiante... de la RAE). Sin embargo, Portolés ([1998] 2001) afirma que esto no se corresponde con la realidad. Propone para probarlo un ejercicio en el que se debe elegir entre los conectores al contrario, por el contrario y en cambio para rellenar varios espacios vacíos en un diálogo. La solución demuestra que estos tres marcadores no son intercambiables en varios contextos, por lo que no son sinónimos (Portolés [1998] 2001, pp. 83-85). Esta posición es la que asume el DPDE que tiene una entrada para cada uno de estos conectores.

El conector por el contrario introduce un miembro discursivo que es contrario al miembro que lo antecede. Tiene no solo el significado de contraste, sino también el de contrariedad (Martín Zorraquino y Portolés, 1999, pp. 4110-4111) o contraposición (Montolío, 2001, p. 85), lo que lo diferencia, por ejemplo, del conector en cambio. También introduce un miembro discursivo que puede refutar el miembro anterior (DPDE, 2008). Señala Montolío que en este último caso el conector introduce un argumento que niega la verdad que se introduce en el primer miembro, y esto sucede porque el conector vincula dos miembros que comentan el mismo tópico y el primero de ellos presenta polaridad negativa (Montolío, 2001, p. 87). También destaca el DPDE (2008) que se documenta su uso en posición final del miembro discursivo y aparece con mayor frecuencia en el registro formal de la lengua.

Por su parte, el conector al contrario introduce, igualmente, un miembro que contradice el miembro discursivo anterior y en muchas ocasiones lo refuta. En Martín 
Zorraquino y Portolés se presentan algunas pruebas que demuestran su no gramaticalización como conector (1999, p. 4112). Sin embargo, se presentan otras en las que su comportamiento se acerca al de los MD y hace que se distinga de gran parte de los adverbios (1999, p. 4113). En esta investigación nos basamos en esos comportamientos que lo acercan a los conectores contraargumentativos y lo consideramos como tal.

Sus propiedades distribucionales, por ejemplo, que puede aparecer como elemento autónomo en un turno de palabra, lo diferencian de por el contrario. Es por ello que lo consideraremos como conector semejante a por el contrario, pero no una variante.

En el $D B E$ se recogen estos conectores como variantes. Véase:

(17) contrario, a adj. [...] // loc. adv. al contrario, por el contrario, de lo contrario. De modo distinto, al revés.

La definición que se ofrece es mixta, es decir, perifrástica ${ }^{13}$ y sinonímica, y en cualquiera de los dos casos, conceptual. Responde al significado de al contrario cuando actúa como locución adverbial y no como conector.

Creemos que los residuos del significado conceptual de la unidad léxica contrario, a en al contrario y por el contrario hace que ambas se definan de forma conceptual, que no se distinga entre el significado de al contrario como locución adverbial y como conector discursivo, y que el significado de procesamiento de por el contrario no se refleje ${ }^{14}$.

\subsection{EL CASO DE POR (LO) TANTO}

Este conector consecutivo se caracteriza por presentar el miembro que introduce como una consecuencia del miembro que lo antecede, derivada de una reflexión, de un razonamiento (Martín Zorraquino y Portolés, 1999, pp. 4100-4101). Se indica en el DPDE que la variante por tanto tiene mayor frecuencia de uso en el registro formal de la lengua.

\footnotetext{
${ }^{13}$ En este caso una definición perifrástica relacional, muy empleada para los adverbios. Para las características de este tipo de definición, véase Porto Dapena (2014, pp. 92-101).

${ }^{14}$ Un diccionario que recoge de cierta forma esta diferencia es el Diccionario de uso del español de María Moliner ([1966-1967] 2008). Véase la entrada contrario, -a.
} 
Su definición en el diccionario escolar que nos ocupa es la siguiente:

(18) tanto, a [...] // loc. conj. por tanto o por lo tanto. Por lo que, en consecuencia.

La definición se aleja de ser funcional o explicativa. Nuevamente este diccionario recurre a una definición sinonímica para apuntar el significado de un conector.

En primer lugar, se define con una construcción (por lo que) que Montolío (2001) clasifica como conector integrado en la oración. Al decir de esta autora, esta expresión conectiva relaciona de manera estrecha la consecuencia que introduce con la causa que la antecede (2001, pp. 105-108). Aun cuando esta construcción conectiva enlace causa y consecuencia al igual que por lo tanto, sus características sintácticas, prosódicas, distribucionales e, incluso, semántico-pragmáticas difieren. Por tal motivo, no constituye un sinónimo de por (lo) tanto y su uso como definición no esclarece el significado de este último.

En segundo lugar, el conector en consecuencia, si bien pertenece al grupo de los consecutivos, se diferencia de por (lo) tanto en que introduce una consecuencia que no deriva de un razonamiento previo. Solo conduce de un estado de cosas, que constituye la causa, al efecto. No así por (lo) tanto, que el hecho de estar fundamentado en un razonamiento hace que se puedan invertir, generalmente, los miembros discursivos que vincula (Martín Zorraquino y Portolés, 1999, pp. 4104-4105; Montolío, 2001, pp. 125-129).

De otro lado, hemos de señalar que la búsqueda del conector en consecuencia en el $D B E$ conduce a una pista perdida, pues este no se encuentra sublematizado.

\subsection{EL CASO DE ENTONCES}

Nos hallamos ante un conector con un débil sentido consecutivo, según señalan Martín Zorraquino y Portolés (1999). Se emplea para dar continuidad al discurso a partir de la presentación de informaciones nuevas que aportan al desarrollo de un tópico general. Estos autores precisan que este sentido laxo de consecuencia se deriva de su originario sentido temporal (Martín Zorraquino y Portolés, 1999, pp. 4107-4109).

El DBE define este conector como sigue:

(19) entonces [...] 2. conj. En consecuencia, por lo tanto. 
Se acude a la definición sinonímica múltiple que nos lleva, por una parte, a la pista perdida que es el conector en consecuencia y, por otra, al conector por lo tanto, que, como se analizó, conduce igualmente a en consecuencia (pista perdida) y a por lo que (falso sinónimo de por (lo) tanto).

Una vez más este diccionario hace que su usuario potencial se enfrente a una serie de remisiones que lo dejan en el total vacío.

\subsection{EL CASO DE PUES}

El conector consecutivo pues se emplea para introducir un miembro discursivo que se presenta como consecuente del miembro discursivo anterior (Martín Zorraquino y Portolés, 1999, p. 4099). Una de las características fundamentales de este conector es que rechaza la posición inicial en el miembro del discurso que introduce y va seguido de pausa ${ }^{15}$. Montolío destaca que es un conector de uso poco frecuente, dado su aspecto formal (2001, p. 118).

Se recoge en el $D B E$ como se muestra a continuación:

(20) pues $[\ldots] 2$ conj. consecutiva. En consecuencia, por lo tanto.

Observamos que la definición es sinonímica y coincide con la presentada para el conector consecutivo entonces ${ }^{16}$. Por una parte, consideramos que estos conectores aun cuando tienen un valor consecutivo no son sinónimos. Por otra, nos llama la atención la falta de coherencia interna del diccionario analizado, pues establece relaciones sinonímicas que no consigna en cada artículo: no se ha empleado la marca Sin. (sinónimo) para relacionar los conectores entonces y pues. Esto nos mueve a pensar que no se ha sido consciente de que ambas unidades léxicas están definidas de igual manera.

\section{CONCLUSIONES}

El diccionario se erige como una herramienta fundamental en el proceso de enseñanza -aprendizaje de una lengua, ya sea materna o extranjera. A él acuden los docentes, los

${ }^{15}$ Cuando esto no ocurre se confunde con el MD estructurador de la información (comentador) pues (Martín Zorraquino y Portolés, 1999, p. 4083; 4100) o con la conjunción causal pues (Montolío, 2001, p. 117). 
escolares y todo aquel que necesite obtener información de diversa naturaleza sobre la lengua (significado de las palabras, aspectos gramaticales, ortográficos, sinónimos y antónimos, etc.). Su compromiso social y el carácter de 'portador de la verdad sobre el significado y uso de las palabras' que se le atribuye conllevan que sea realizado con el mayor rigor científico.

$\mathrm{El}$ análisis realizado reveló algunas inconsistencias internas del $D B E$ con respecto al tratamiento de las definiciones de los conectores discursivos, así como incongruencias con postulados teóricos actuales. A continuación resumiremos las más relevantes:

- No se emplea en ningún caso la definición funcional o explicativa, que es el desiderátum en cuanto a tipo de definición para las palabras gramaticales.

- En todos los casos se emplean definiciones de tipo sinonímico, aunque realmente lo que encontramos son falsos sinónimos (los definidores no son auténticos sinónimos de los definidos); por lo que las definiciones se pueden clasificar de parasinonimicas, siguiendo la terminología de Porto Dapena $(2002,2014)$.

- El empleo de las definiciones antes mencionadas condujo a problemas infraestructurales en el diccionario como la circularidad y las pistas perdidas.

Lo antes expuesto conduce a pensar en la necesidad de redefinir estas unidades lingüísticas en el $D B E$, a partir del empleo de definiciones sencillas, de tipo funcional, y despojadas de un metalenguaje complejo; en aras de que los escolares, destinatarios potenciales de esta obra lexicográfica, comprendan el significado y la función de este tipo de unidades léxicas, y puedan utilizarlas de manera correcta y efectiva. Cerramos con el siguiente ejemplo, a modo de propuesta:

(21) contrario, a. adj. [...] // al contrario. loc. adv. De modo distinto. EJEMPLO ${ }^{17} /$ nexo con valor adversativo. Se utiliza para indicar contrariedad con la información anterior o refutarla. EJEMPLO. (Puede ir seguido o no de la información que indica cómo debe interpretarse este sentido de contrariedad en relación con lo dicho anteriormente. EJEMPLO. En el diálogo, puede aparecer de manera independiente. EJEMPLO). Af. por el contrario. // por el contrario. loc. adv. nexo con valor adversativo. Se utiliza para introducir una información contraria a la información anterior o refutarla. EJEMPLO. Ú. m. en el registro formal de la lengua. Af. al contrario.

\footnotetext{
${ }^{17}$ Se ha mantenido este significado para que quede más clara la posición que adoptamos con respecto a los cambios que creemos han de efectuarse en este artículo lexicográfico.
} 


\section{BIBLIOGRAFÍA}

Azorín Fernández, D. (2000). Los diccionarios didácticos del español desde la perspectiva de sus destinatarios, ELUA. Estudios de Linguiistica Universidad de Alicante, 14, pp. 19-44.

Blakemore, D. (1987). Semantic constraints on relevance. Oxford: Blackwell.

Briz Gómez, A. (1993). Los conectores pragmáticos en español coloquial (I): su papel argumentativo, Contextos, XI, 21/22, pp. 145-188.

Briz, A., Pons, S. y J. Portolés (Coords.) (2008). Diccionario de partículas discursivas del español [en línea] $\leq$ www.dpde.es $>$

Cuartero Sánchez, J. M. (1995). El estatuto categorial de además y sus propiedades distribucionales, Dicenda, 13, pp. 103-118.

Forgas, E. y M. Bargalló (2005-2006). Los diccionarios del Centro de Lingüística Aplicada de Santiago de Cuba, Revista de Lexicografía, XII, pp. 249-266.

Fuentes Rodríguez, C. (1996). La sintaxis de los relacionantes supraoracionales. Madrid: Arco Libros.

Fuentes Rodríguez, C. (2003). Operador/conector, un criterio para la sintaxis discursiva, RILCE, 19.1, pp. 61-85.

Fuentes Rodríguez, C. (2009). Diccionario de conectores y operadores del español. Madrid: Arco Libros.

García Roche, A. de la C. (2018). Los marcadores conversacionales enfocadores de la alteridad en una muestra del español coloquial de La Habana, Universidad de La Habana, 286, pp. $72-81$ [en línea] http://scielo.sld.cu/scielo.php?script=sci arttext\&pid=S025392762018000200005\&lng=es\&tlng=es.

Gili y Gaya, S. (1966 [1943]). Curso superior de sintaxis española. La Habana: Edición Revolucionaria.

González Mafud, A. M. y M. Perdomo Carmona (2014). Marcadores del discurso de La Habana, Cuadernos de la ALFAL, 5, pp. 107-139.

Hernández Hernández, H. (1989). Los diccionarios de orientación escolar. Contribución al estudio de la lexicografía monolingüe española. Tubinga: Max Niemeyer Verlag.

Hernández Hernández, H. (2003). La lexicografía escolar del español: desde sus orígenes hasta su consolidación, Revista Internacional de Lingüistica Iberoamericana, I, pp. 7-25.

Hernández Hernández, H. (2008). Retos de la lexicografía didáctica española. En Azorín Fernández D. et al. (Eds.), El diccionario como puente entre las lenguas y las culturas del mundo. Actas del II Congreso Internacional de Lexicografía Hispánica. Alicante: Fundación Biblioteca Virtual Miguel de Cervantes, pp. 22-32.

Holgado Lage, A. (2014). Pragmática lingüística aplicada al español para extranjeros, con especial atención a los marcadores discursivos: El Diccionario de Marcadores Discursivos para Estudiantes de Español como Lengua Extranjera, Tesis doctoral dirigida por Elena Bajo Pérez, Facultad de Filología, Universidad de Salamanca [en línea] http://hdl.handle.net/10366/132282. 
Holgado Lage, A. (2018). Diccionario de Marcadores Discursivos para estudiantes de español como segunda lengua. Berlin / New York: Peter Lang.

Llopis Cardona, A. (2006). Aplicación de la teoría de Rey-Debove a las definiciones lexicográficas de los marcadores discursivos. En M. Villayandre Llamazares (Ed.), Actas del XXXV Simposio Internacional de la Sociedad Española de Lingüistica. León: Universidad de León, Departamento de Filología Hispánica y Clásica. pp. 1086-1105

López Serena, A. y M. Borreguero Zuloaga (2010). Los marcadores del discurso y la variación lengua hablada vs. lengua escrita. En O. Loureda Lamas y E. Acín-Villa (Coords.), Los estudios sobre marcadores del discurso en español, hoy. Madrid: Arco Libros, pp. 325-405.

Maldonado González, C. (Dir.) (2003). Diccionario Secundaria y Bachillerato. Lengua Española, (2. ${ }^{a}$ ed.). Madrid: Ediciones SM.

Martín Zorraquino, M. ${ }^{a}$ A. (2004). El tratamiento lexicográfico de los marcadores del discurso y la enseñanza de E/LE. En M.a A. Castillo Carballo (Coord.), Actas del XV Congreso Internacional de ASELE, pp. 53-67.

[en línea] http://cvc.cervantes.es/ensenanza/biblioteca ele/asele/pdf/15/15 0051.pdf.

Martín Zorraquino, M. a A. y J. Portolés Lázaro (1999). Los marcadores del discurso. En I. Bosque y V. Demonte (Dirs.), Gramática descriptiva de la lengua española. Madrid: Espasa Calpe, 3 Vols. pp. 40514213.

Martínez Abreu, Y. (2015). Glosar el marcador discursivo bueno en el español coloquial de La Habana. En IV Coloquio Internacional Marcadores del discurso en las lenguas románicas. Universidad de Heidelberg (Alemania).

Miyares Bermúdez, E. et al. (2006). Léxico activo-funcional del escolar cubano. Santiago de Cuba: Centro de Lingüística Aplicada.

Miyares Bermúdez, E. (Dir.) (2014). Diccionario básico escolar (4. ${ }^{\text {a }}$ ed.). Santiago de Cuba: Editorial Oriente. Moliner, M. (1966-1967). Diccionario de uso del español, versión electrónica 3.0. Madrid: Gredos.

Montolío Durán, E. (2001). Conectores de la lengua escrita. Barcelona: Ariel.

Nomdedeu Rull, A. (2012). Miyares Bermúdez, Eloína (Dir.) (2009): Diccionario básico escolar. Santiago de Cuba, Centro de Lingüística Aplicada, Revista de Lexicografía, XVII, pp. 243-256.

Perdomo Carmona, M. (2013). Estudio de los marcadores discursivos conversacionales en el habla culta de La Habana, en Memorias. VIII Conferencia Internacional Lingüistica 2013 (CD-ROM). La Habana: Instituto de Literatura y Lingüística.

Pons Bordería, S. (1998). Conexión y conectores, Estudio de su relación en el registro informal de la lengua. Valencia: Universitat de València.

Porto Dapena, J. Á. (2002). Manual de técnica lexicográfica. Madrid: Arco Libros.

Porto Dapena, J. Á. (2014). La definición lexicográfica. Madrid: Arco Libros.

Portolés Lázaro, J. (2001). Marcadores del discurso (2. ${ }^{a}$ ed.). Barcelona: Ariel. 
Real Academia Española y Asociación de Academias de la Lengua Española. (2009). Nueva gramática de la lengua española. Madrid: Espasa.

Real Academia Española. (2011). Diccionario del estudiante. Secundaria y Bacbillerato (2. ${ }^{a}$ ed.). Madrid: Santillana Ediciones Generales.

Real Academia Española. (2014). Diccionario de la lengua española (23. ${ }^{a}$ ed.). Madrid: Espasa-Calpe.

Ruiz Miyares, L. y S. Tarp (2015). Los diccionarios escolares cubanos: una experiencia para compartir. Estudios de Lexicografia, 4, pp. 185-198.

Sánchez Muñoz, T. (2002-2003). Los diccionarios escolares a comienzos del siglo XXI, Revista de Lexicografia, IX, pp. 175-188.

Sanmartín Sáez, J. (2010). La dificultad de definir en un diccionario de partículas. En M. ${ }^{a}$ A. Castillo Carballo y J. M. García Platero (Coord.), La lexicografía en su dimensión teórica. Málaga: Universidad de Málaga. pp. 561-585.

Santos Río, L. (2003). Diccionario de particulas. Salamanca: Luso-Española.

Schiffrin, D. (1987). Discourse markers. Cambridge: Cambridge University Press.

Seco, M. (1987). Problemas formales de la definición. En Estudios de lexicografía Española. Madrid: Gredos. pp. 15-34.

Vázquez Veiga, N. (1995-96). Los marcadores discursivos en las obras lexicográficas, Revista de Lexicografia, 2, pp. 133-149.

Vázquez Veiga, N. (2000). Los marcadores discursivos en el Diccionario de colocaciones y marcadores del español. En U. Heid et al. (Eds.), Proceedings of the Ninth euralex International Congress. Stuttgart: $\begin{array}{llllll}\text { Universität } & \text { Stuttgart. } & \text { pp. } & 761-771 & \text { línea] }\end{array}$ <http://www.euralex.org/elx proceedings/Euralex2000/087 Nancy\%20VAZQUEZ\%20VE IGA Los $\% 20$ marcadores $\% 20$ discursivos $\% 20$ en $\% 20$ el $\% 20$ Diccionario $\% 20$ de $\% 20$ colocaciones $\% 20 \mathrm{y} \% 20$ marcadores $\% 20$ del $\% 20$ espanol.pdf $>$.

Vázquez Veiga, N. (2008). Las etiquetas discursivas en un diccionario de marcadores pragmáticos: Un puente entre lenguas. En D. Azorín Fernández et al. (Coords.), El diccionario como puente entre las lenguas y culturas del mundo: actas del II congreso Internacional de Lexicografía Hispánica [en línea] $\leq$ http://media.cervantesvirtual.com/s3/BVMC OBRAS/020/6b1/768/2b2/11d/fac/c70/0 21/85c/e60/64/mimes/0206b176-82b2-11df-acc7-002185ce6064.pdf>. 